\title{
Über die chemische Zusammensetzung und Konstitution des Vesuvians.
}

\author{
Von \\ P. JanNASCh und P. Weingarten.
}

Die im nachstehenden mitgeteilten neun Vesuviananalysen sind vor mehreren Jahren auf Veranlassung und unter Leitung des einen von uns durch H. Voges ausgeführt und in dessen InauguralDissertation ${ }^{1}$ veröffentlicht worden. Wir haben damals die ersten Wasserbestimmungen nach der Bleichromatmethode ${ }^{2}$ unternommen, hatten aber damals noch nicht die Erfahrung gemacht, dafs man das Wasser erst durch andauerndes Zusammenschmelzen der betreffenden Mischung vollständig auszutreiben imstande ist, welches Verhalten unmittelbar darauf bei der Ausführung einer Reihe vollständiger und genauer Turmalinanalysen ${ }^{3}$ erkannt wurde. Mit Hilfe unserer neuesten Boraxschmelzmethode ${ }^{4}$ haben wir nun den Wassergehalt des Vesuvians mit aller Sicherheit und Schärfe ermittelt. Gleichzeitig wurden auch einige der früheren wichtigen Bestimmungen, wie diejenigen des Fluors, des Eisenoxyduls etc. einer Kontrolle unterzogen, ohne dal's wir hierbei auf irgendwie in Betracht kommende Abweichungen gestolsen wären. Nach unseren vollständigen Analysen kommen im Vesuvian zeln bis zwölf verschiedene Körper vor und nicht blols fünf bis sechs, welche die meisten älteren Analysen aufweisen. Von besonderer Wichtigkeit ist die Thatsache, dals eine Reihe von Vesuvianen Fluor enthält, während andere vollständig frei davon sind, d. h. sich in einem Zustande der Wandelbarkeit befinden, in welchem bereits alles Fluor durch Hydroxyl ersetzt ist. ${ }^{5}$ Weiterhin lülst sich nicht leugnen, dal's das Eisen teilweise in der Form von Oxyd vorhanden ist, was uns sämtliche nach verschiedenen Methoden wiederholt ausgeführte Eisenoxydulbestimmungen bestätigten. Gleichzeitig vorhanden sind endlich kleine Mengeu von Mangan, Kalium, Natrium und Lithium, selbst bei den reinsten Varietäten. Auch seltenere Elemente, wie

1 Über die chem. Zusammensetsung des Veswvians, Göttingen (1887), bei E. A. Hutiı.

2 Ber. deutsch. chem. Ges. 22, 221.

3 Ber. deutsch. chem. Ges. 22, 216.

4 Diese Zritschir. 8, 352.

${ }^{5}$ Cf. Diese Zeitschr. 6, 325 und 7, 915. 
das Titan, fehlen in dem Vesuvian nicht, und mlängst fanden wir in einem braunen, auffallend dunklen Krystall vom Vesuv, dessen volle Analyse wir später mitteilen werden, fast $4 \%$ davon. Der Borsäuregehalt des Wiluits ist von dem einen von uns vor nun 10 Jahren aufgefunden und zu annähernd $3 \%$ festgestellt worden (eine spätere Analyse von Rammelsberg ergab 2.54\% Borsäure). ${ }^{1}$

\section{Zusammenstellung der analytischen Resultate.}

I. Fluorfreie Vesuviane.

Vesuvian von Zermatt.

Gefunden: Berechnet:

$$
\text { Arquiv: }
$$

\begin{tabular}{|c|c|c|c|c|c|c|c|c|}
\hline $\begin{array}{l}\mathrm{SiO}_{2} \\
\mathrm{TiO}_{2}\end{array}$ & $\begin{array}{r}37.49 \\
1.20\end{array}$ & $\begin{array}{l}= \\
=\end{array}$ & $\begin{array}{r}37.21 \\
1.19\end{array}$ & $\begin{array}{l}=\mathrm{Si} \\
=\mathrm{Ti}\end{array}$ & $\begin{array}{r}17.3879 \\
0.7367\end{array}$ & $\begin{array}{l}= \\
=\end{array}$ & $\begin{array}{l}0.6210 \\
0.0147\end{array}$ & $0.6357=1.90$ \\
\hline $\begin{array}{l}\mathrm{Fe}_{2} \mathrm{O}_{3} \\
\mathrm{Al}_{2} \mathrm{O}_{3}\end{array}$ & $\begin{array}{r}4.68 \\
14.74\end{array}$ & $\begin{array}{l}= \\
=\end{array}$ & $\begin{array}{r}4.65 \\
14.63\end{array}$ & $\begin{array}{l}=\mathrm{III} \\
=\mathrm{Al}\end{array}$ & $\begin{array}{l}3.2804 \\
7.7598\end{array}$ & $\begin{array}{l}= \\
=\end{array}$ & $\begin{array}{l}0.0587 \\
0.2870\end{array}$ & $0.3457=1.03$ \\
\hline $\mathrm{FeO}$ & 1.09 & $=$ & 1.08 & $=\mathrm{Fe}$ & 0.8401 & $=$ & 0.0150 . & \multirow{4}{*}{$0.7046=2.10$} \\
\hline $\mathrm{CaO}$ & 35.43 & $=$ & 35.17 & $=\mathrm{Ca}$ & 25.1232 & $=$ & 0.6295 & \\
\hline $\mathrm{MnO}$ & Spuren & $=$ & - & $=\mathrm{Mn}$ & - & $=$ & - & \\
\hline $\mathrm{MgO}$ & 2.42 & $=$ & 2.40 & $=M_{g}$ & 1.4400 & $=$ & 0.0601 & \\
\hline $\mathrm{K}_{2} \mathrm{O}$ & 0.66 & $=$ & 0.66 & $=\mathbf{K}$ & 0.5480 & $=$ & 0.0140 & \multirow{3}{*}{$0.3349=1.00$} \\
\hline $\mathrm{Na}_{2} \mathrm{O}$ & 0.18 & $=$ & 0.18 & $=\mathrm{Na}$ & 0.1336 & $=$ & 0.0058 & \\
\hline $\mathrm{H}_{2} \mathrm{O}$ & 2.85 & $=$ & 2.83 & $=\mathrm{H}$ & 0.3151 & $=$ & 0.3151 & \\
\hline & 100.74 & & 100.00 & & 57.5648 & & & \\
\hline
\end{tabular}

Vesuvian von Corbassera (Ala).

Gefunden: Berechnet:

Äquiv.:

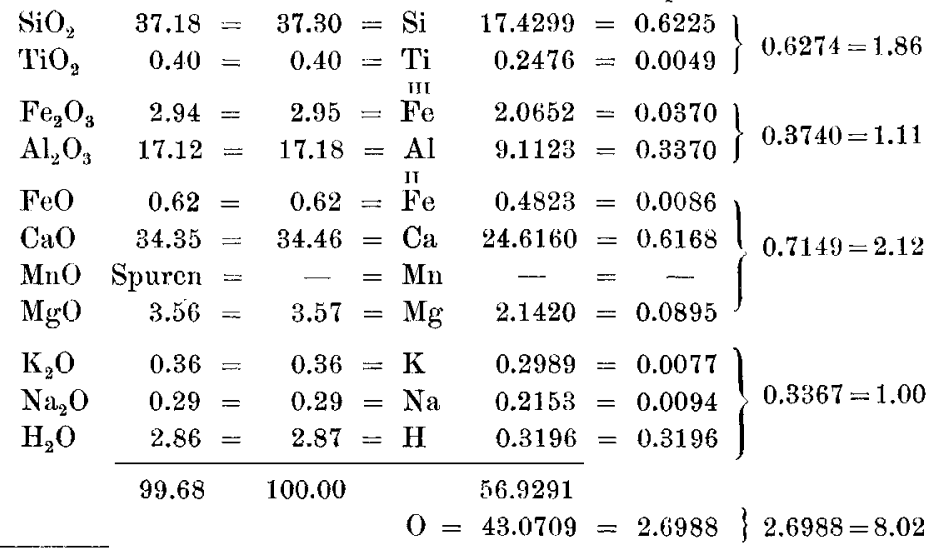

1 Neues Jahrb. Miner. (1884) 1, 270 und Rammelsbera, Mineralchemie (Ergänzungsheft zur 2. Aufl.), S. 262. - Hinsichtlich des von uns benutzten ana- 


\section{Vesuvian von Csiklova.}

Gefunden: Berechnet:

Äquiv.:

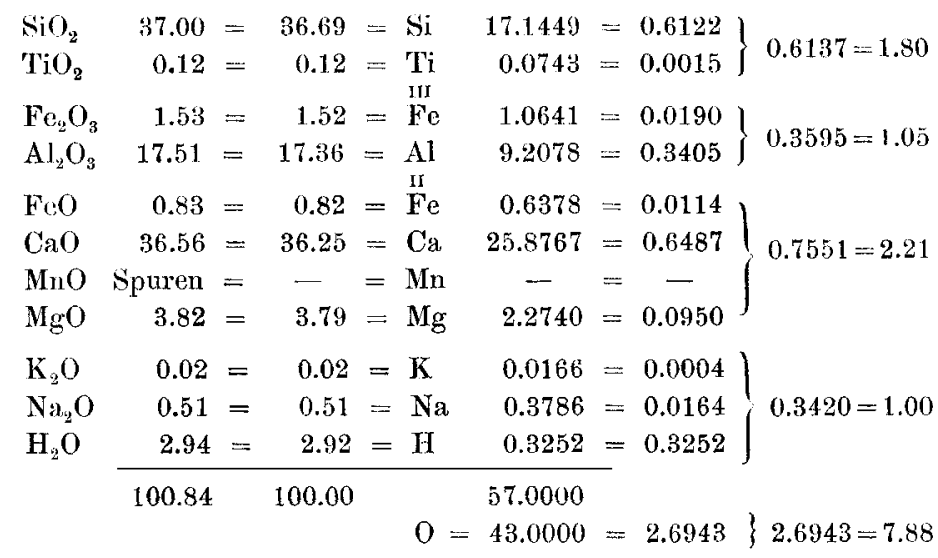

Vesuvian von Canzocoli.

Gefunden: Berechnet: $\quad \ddot{A}_{\text {quiv.: }}$

\begin{tabular}{|c|c|c|c|c|c|c|c|c|}
\hline $\begin{array}{l}\mathrm{SiO}_{2} \\
\mathrm{TiO}_{2}\end{array}$ & $\begin{array}{c}36.29 \\
-\end{array}$ & $\begin{array}{l}= \\
=2\end{array}$ & $\begin{array}{c}36.10 \\
-\end{array}$ & $\begin{array}{l}=\mathrm{Si} \\
=\mathrm{Ti}\end{array}$ & $\begin{array}{c}16.8692 \\
\ldots\end{array}$ & $=$ & $\begin{array}{l}0.6025 \\
--\end{array}$ & $0.6025=1.81$ \\
\hline $\mathrm{Fe}_{2} \mathrm{O}_{3}$ & 3.85 & $=$ & 3.83 & $=\stackrel{\mathrm{I}^{\prime \prime \prime}}{\mathrm{F}}$ & 2.6813 & $=$ & 0.0480 & \multirow{2}{*}{$0.3662=1.10$} \\
\hline $\mathrm{Al}_{2} \mathrm{O}_{3}$ & 16.31 & $=$ & 16.22 & $=\mathrm{Al}$ & 8.6032 & $=$ & 0.3182 & \\
\hline FeO & 1.23 & $=$ & 1.22 & $=F_{0}$ & 0.9490 & $=$ & 0.0170 & \multirow{4}{*}{$0.7253=2.18$} \\
\hline $\mathrm{CaO}$ & 36.01 & $=$ & 35.82 & $=\mathrm{Ca}$ & 25.5862 & $=$ & 0.6411 & \\
\hline $\mathrm{MnO}$ & - & $=$ & - & $=\mathrm{Mn}$ & - & & - & \\
\hline $\mathrm{MgO}$ & 2.70 & $=$ & 2.68 & $=\mathrm{Mg}$ & 1.6080 & $=$ & 0.0672 & \\
\hline \multirow{5}{*}{$\begin{array}{l}\mathrm{K}, \mathrm{O} \\
\mathrm{N}_{\mathrm{l}_{2}} \mathrm{O} \\
\mathrm{H}_{2} \mathrm{O}\end{array}$} & 0.23 & $=$ & 0.23 & $=\mathrm{K}$ & 0.1902 & $=$ & 0.0051 & \multirow{3}{*}{$0.3327=1.00$} \\
\hline & 1.36 & $=$ & 1.35 & $=\mathrm{Na}$ & 1.0022 & $=$ & 0.0436 & \\
\hline & 2.56 & $=$ & 2.55 & $=\mathrm{H}$ & 0.2840 & $=$ & 0.2840 & \\
\hline & 100.54 & & 100.00 & & 57.7733 & & & \\
\hline & & & & & 42.2267 & $=$ & 2.6458 & $2.6458=7.95$ \\
\hline
\end{tabular}

lytischen Tremungsganges verweise ich hier auf meine frühere Untersuchung des Vesuvians vom Vesuv (Neues Jahrb. Min. $\lfloor 1883 \mid$ 2, 123), auf diejenige des Axinits von Bourg d'Oisans (Diese Zeitschr. 6, 57) und auf den von mir ausgearbeiteten, ganz kürzlich von M. Dтттricr publizierten Gang der Gesteinsanalyse (Mitteil. d. Gro/sh. Bad. Geol. Landesanstalt $\lfloor 3\rfloor 3,77-106$ ). 


\section{Fluorhaltige Vesuviane.}

Vesuvian von Sandford.

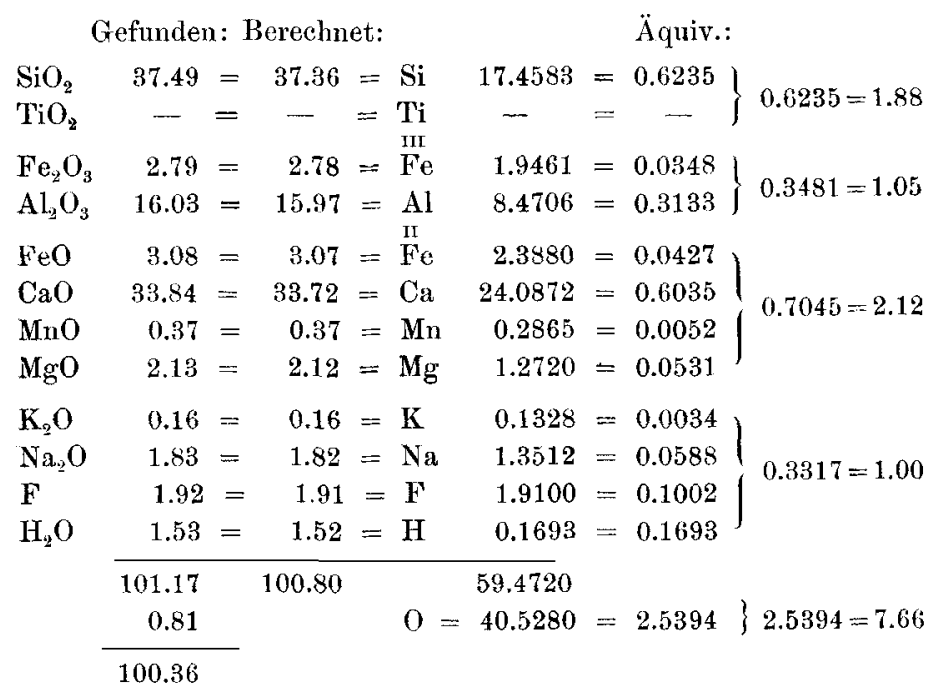

Vesuvian von Egg.

Gefunden: Berechnet:

Äquiv.:

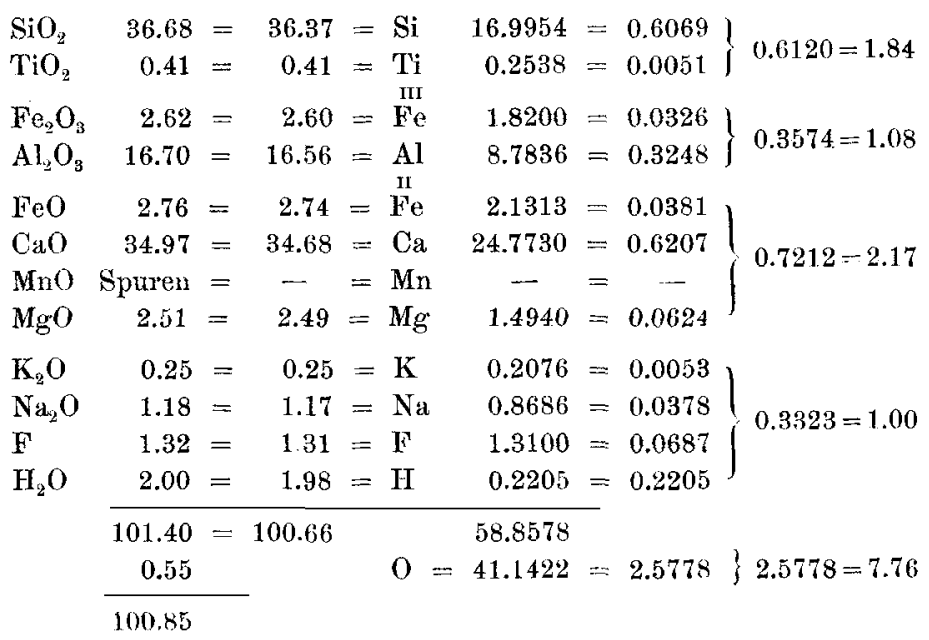


Vesuvian von Arendal.

\begin{tabular}{|c|c|c|c|c|c|c|}
\hline \multicolumn{4}{|c|}{ Gefunden: Bereehnet: } & \multicolumn{3}{|c|}{$\ddot{A}_{\text {(quiv.: }}$} \\
\hline $\mathrm{SiO}_{2}$ & 36.81 & $=\quad 36.27$ & $=\mathrm{si}$ & 16.9489 & $=0.6053$ & \\
\hline & 0.28 & 0.28 & $=\mathrm{Ti}$ & 0.1733 & $=0.0035$ & $0.6080=1.83$ \\
\hline $\begin{array}{l}\mathrm{Fe}_{2} \mathrm{O}_{3} \\
\mathrm{Al}_{2} \mathrm{O}_{3}\end{array}$ & $\begin{array}{r}3.92 \\
16.25\end{array}$ & $\begin{array}{l}=3.86 \\
=16.01\end{array}$ & & & $\begin{array}{l}=0.0484 \\
=0.3140\end{array}$ & $0.3624=1.09$ \\
\hline $\mathrm{FeO}$ & 2.21 & $=2.18$ & $=\stackrel{\mathrm{II}}{\mathrm{Fe}}$ & 1.6957 & $=0.0303$ & \\
\hline $\mathrm{CaO}$ & 35.49 & $=34.97$ & $=\mathrm{Ca}$ & 24.9806 & $=0.6259$ & \\
\hline $\mathrm{MnO}$ & 0.14 & $=0.14$ & $=M_{1}$ & 0.1084 & $=0.0020$ & $0.7254=2.18$ \\
\hline MgO & 2.72 & 2.68 & $=\mathrm{Mg}$ & 1.6080 & $=0.0672$ & \\
\hline $\mathrm{K}_{2} \mathrm{O}$ & 0.16 & 0.16 & $=\mathbf{K}$ & 0.1328 & $=0.0034$ & \\
\hline $\mathrm{Na}_{2} \mathrm{O}$ & 0.52 & 0.51 & $=\mathrm{N}_{\mathrm{i}}$ & 0.3786 & $=0.0165$ & \\
\hline $\mathbf{F}$ & 1.36 & 1.34 & $=\mathbf{F}$ & 1.3400 & $=0.0703$ & $0.3330=1.00$ \\
\hline $\mathrm{H}_{2} \mathrm{O}$ & 2.21 & 2.18 & $=\mathrm{H}$ & 0.2428 & $=0.2428$ & \\
\hline & 102.07 & $=100.58$ & $=$ & 58.8032 & & \\
\hline & 0.56 & & & 41.1968 & $=2.5813$ & $2.5813=7.75$ \\
\hline
\end{tabular}

Vesuvian von Egeran (Haslau).

Gefunden: Berechnet:

Äquiv.:

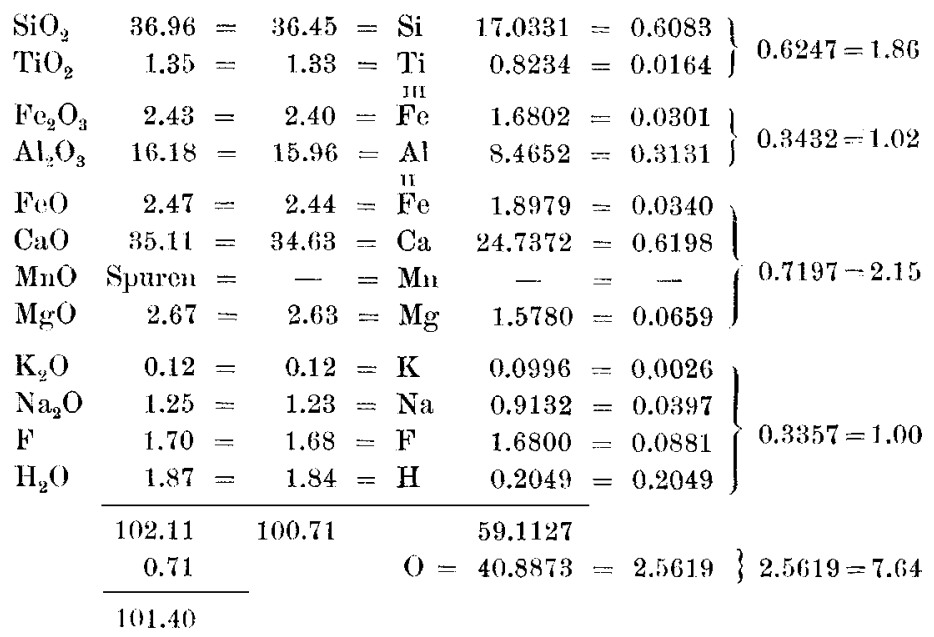


Vesuvian von Eker.

\begin{tabular}{|c|c|c|c|c|c|c|c|}
\hline \multicolumn{4}{|c|}{ Gefunden: Berechmet: } & \multicolumn{4}{|c|}{ Äquiv.: } \\
\hline $\mathrm{SiO}_{2}$ & 36.92 & $=36.62$ & $=\mathrm{Si}$ & 17.1124 & $==$ & 0.6112 & \multirow[b]{2}{*}{$0.6220=1.85$} \\
\hline $\mathrm{TiO}_{2}$ & 0.89 & 0.88 & $=\mathrm{T} \mathrm{i}$ & 0.5448 & $=$ & 0.0108 & \\
\hline $\mathrm{Fe}_{2} \mathrm{O}_{3}$ & 3.46 & 3.42 & $=\mathrm{Fc}^{\mathrm{III}}$ & 2.3943 & $=$ & 0.0428 & \multirow[b]{2}{*}{$0.3423=1.02$} \\
\hline $\mathrm{Al}_{2} \mathrm{O}_{3}$ & 15.43 & 15.27 & $=\mathrm{Al}$ & 8.0993 & $=$ & 0.2995 & \\
\hline $\mathrm{FeO}$ & 1.51 & 1.49 & $=\mathrm{Fe}$ & 1.1590 & $=$ & 0.0207 & \multirow{4}{*}{$0.7304=2.17$} \\
\hline $\mathrm{CaO}$ & 35.81 & 35.44 & $=\mathrm{Ca}$ & 25.3159 & $=$ & 0.6343 & \\
\hline $\mathrm{MnO}$ & Spuren & - & $=\mathrm{Mn}$ & - & $=$ & - & \\
\hline $\mathrm{MgO}$ & 3.04 & 3.01 & $=\mathrm{Mg}$ & 1.8060 & $=$ & 0.0754 & \\
\hline $\mathrm{K}_{2} \mathrm{O}$ & 0.18 & 0.18 & $=\mathbf{K}$ & 0.1494 & $=$ & 0.0038 & \multirow{4}{*}{$0.3367=1.00$} \\
\hline $\mathrm{Na}_{2} \mathrm{O}$ & 0.81 & 0.80 & $=\mathrm{Na}$ & 0.5939 & $=$ & 0.0258 & \\
\hline $\mathrm{F}$ & 1.35 & 1.34 & $=\mathbf{F}$ & 1.3400 & $=$ & 0.0703 & \\
\hline \multirow[t]{4}{*}{$\mathrm{H}_{2} \mathrm{O}$} & 2.15 & 2.13 & $=\mathrm{H}$ & 0.2372 & $=$ & 0.2372 & \\
\hline & 101.62 & 100.58 & \multirow{3}{*}{$0=$} & 58.7522 & & & \multirow{3}{*}{$2.5845=7.67$} \\
\hline & 0.56 & & & 41.2478 & $=$ & 2.5845 & \\
\hline & & & & & & & \\
\hline
\end{tabular}

Eine Zusarnmenstellung der den Vesurian bildenden Elemente ergiebt nach den vorstehenden Analysen folgende Verhältnisse:

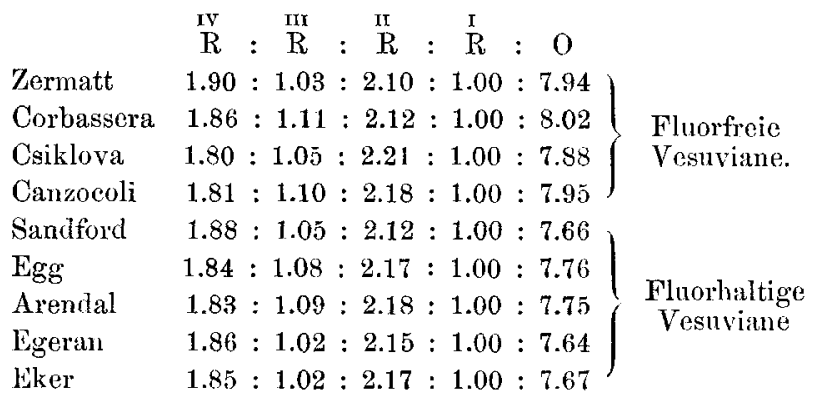

Zur Erläuterung der Berechnung ist hier noch anzugeben, dats die Analysenergebnisse der fluorfreien Vesuviane auf $100 \%$ reduziert wurden. Bei den fluorhaltigen Vesuvianen mulste zunächst die dem gefundenen Fluor entsprechende Sauerstoffmenge in Abzug gebracht werden $\left(\mathrm{F}_{2}: \mathrm{O}=1.92 \% \mathrm{Fl}\right.$ [Vorkommnis von Sandford]: $\mathrm{X}=0.81 \%$; mithin $101.17 \%-0.81=100.36 \%$ ), ehe die Beziehung der Einzelbestimmungen auf 100 nach der Gleichung $100.36: 100=37.49 \mathrm{SiO}_{2}: \mathrm{X}$ $=37.36 \%$ erfolgte. 
Die optischen und krystallographischen Verhältnisse der von uns analysierten Vorkommnisse wird H. v. KraATZ näher studieren und darüber später berichten.

Die Durchschnittsverhältnisse der den Vesurian zusammensetzenden Elemente sind demnach bei den fluorfreien Varietäten

$$
\begin{aligned}
& \stackrel{\mathrm{IV}}{\mathrm{R}}: \stackrel{\mathrm{III}}{\mathrm{R}}: \stackrel{\mathrm{II}}{\mathrm{R}}: \stackrel{\mathrm{I}}{\mathrm{R}}: 0 \\
& 1.85: 1.06: 2.16: 1.00: 7.97
\end{aligned}
$$

und bei den fluorhaltigen Vorkommnissen

$$
1.85: 1.06: 2.16: 1.00: 7.71 \text {. }
$$

Der Sauerstoff in den fluorfreien Vesuvianen ergiebt sich $1: 8$, auf $\stackrel{\mathrm{I}}{\mathrm{R}}$ bezogen. In den fluorhaltigen Verbindungen ist die Sauerstoftmenge entsprechend dem Gehalte an Fluor bei der Berechnung gekürzt worden, so dafs bei der vorstehenden Zusammenstellung die Sauerstoffmenge bei diesen Vesuvianen geringer als bei dem fluorfreien Mineral erscheint.

Die abgerundeten Sauerstoffquotienten liefern das endgültige Verhältnis

$$
\begin{aligned}
& \text { Si }: \stackrel{\text { III }}{R}: \stackrel{\text { II }}{R}: \stackrel{I}{R}: 0 \\
& 2^{\prime}: 1: 2: 1: 8
\end{aligned}
$$

welches für den fluorfreien Vesuvian zu der einfachen Orthosilikatformel

$$
\begin{aligned}
\mathrm{Si}_{2} \mathrm{O}_{8} \stackrel{\text { III II I }}{R} \mathrm{R}_{2} \mathrm{R} & =\left(\mathrm{SiO}_{4}\right)_{2} \cdot \mathrm{Al}(\mathrm{Fe} \cdot \mathrm{Ti}) \cdot \mathrm{Ca}_{2}\left(\mathrm{Mg}_{2} \cdot \mathrm{Fe}_{2} \cdot \mathrm{Mn}_{2}\right) \cdot \mathrm{H}(\mathrm{K} \cdot \mathrm{Na} \cdot \mathrm{Si}) \\
& =\left(\mathrm{SiO}_{4}\right)_{2} \cdot \mathrm{AlCa}_{2} \mathrm{H}
\end{aligned}
$$

führt, resp. zu der Verdoppelung

$$
\mathrm{Si}_{4} \mathrm{O}_{14} \mathrm{Al}_{2} \mathrm{Ca}_{4}(\mathrm{OH})_{2} \text {. }
$$

Graphisch zum Ausdruck gebracht, erhält man die nachstehenden Strukturformeln:

verdoppelt:

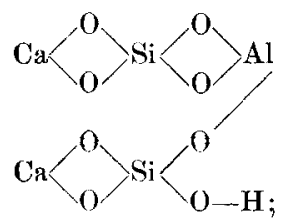

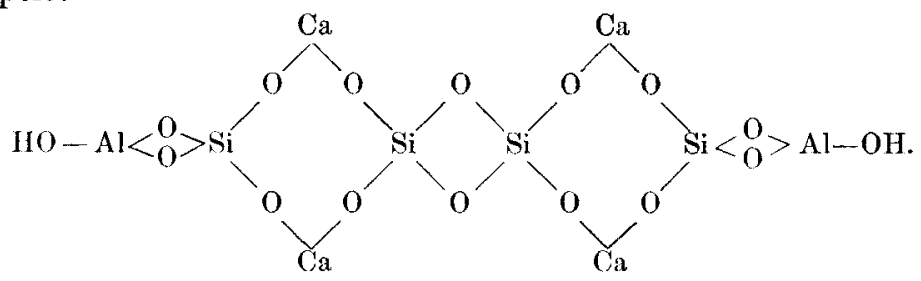


Bei den fluorhaltigen Vesuvianen gewährt die verdoppelte Formel den besten Einblick in dessen Konstitutionsverhältnisse:

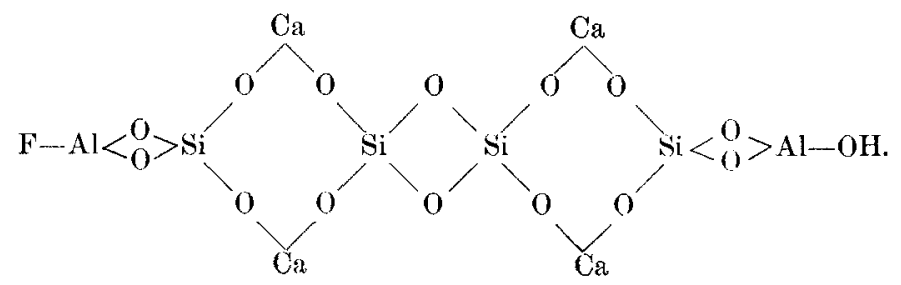

Heidelberg, Universitäts-Laboralorium, Februer 1895.

Bei der Redaktion eingegangen am 25. Februar 1895. 